\title{
Desmoplastic/nodular medulloblastoma associated with anhidrotic ectodermal dysplasia
}

\author{
Yosuke Watanabe $\cdot$ Fumiyuki Yamasaki $\cdot$ Kazuhiro Nakamura Yoshinori Kajiwara $\cdot$ Takeshi Takayasu • \\ Takanori Hirose $\cdot$ Vishwa Jeet Amatya $\cdot$ Kazuhiko Sugiyama $\cdot$ Masao Kobayashi $\cdot$ Kaoru Kurisu
}

Received: 15 September 2012/Accepted: 15 January 2013/Published online: 1 February 2013

(C) The Japan Society of Clinical Oncology 2013

\begin{abstract}
We encountered a 1-year-old boy with desmoplastic/nodular medulloblastoma (DNMB) associated with anhidrotic ectodermal dysplasia (AED). He was admitted to our institute because of ingravescent vomiting. On physical examination, a depressed nasal bridge, low-set ears, thick lips with peg-shaped teeth, hypohidrosis, sparse hair, thin atrophic skin, scaly dermatitis with frontal bossing, enlarged head size, and bulging anterior fontanel were observed. Neuroradiological examination revealed multiple cerebellar masses with heterogeneous enhancement and speckled calcifications. Severe obstructive hydrocephalus was also observed. He underwent surgery based on histologically diagnosed DNMB, and received postoperative multiple-drug chemotherapy with a completely favorable outcome. One year has passed and the disease-free patient is still doing well without any unwanted
\end{abstract}

Y. Watanabe $(\bowtie) \cdot$ F. Yamasaki · Y. Kajiwara · T. Takayasu · K. Kurisu

Department of Neurosurgery, Hiroshima University,

Graduate School of Biomedical and Health Sciences,

1-2-3 Kasumi, Minami-ku, Hiroshima 734-8551, Japan

e-mail: yowatanabe@hiroshima-u.ac.jp

K. Nakamura · M. Kobayashi

Department of Pediatrics, Hiroshima University, Graduate

School of Biomedical and Health Sciences, Hiroshima, Japan

T. Hirose

Department of Diagnostic Pathology, Tokushima Prefectural

Central Hospital, Tokushima, Japan

V. J. Amatya

Department of Pathology, Hiroshima University, Graduate

School of Biomedical and Health Sciences, Hiroshima, Japan

K. Sugiyama

Department of Clinical Oncology and Neuro-oncology Program, Cancer Treatment Center, Hiroshima University Hospital,

Hiroshima, Japan events. AED was diagnosed on the basis of the clinical findings: this is probably the first report on AED-associated DNMB.

Keywords Desmoplastic/nodular medulloblastoma • Anhidrotic ectodermal dysplasia $\cdot$ Hh-signaling

\section{Introduction}

Medulloblastoma, the most common malignant brain tumor in children, accounts for approximately $20 \%$ of all intracranial tumors in patients of less than 20 years of age [1]. Although medulloblastoma is usually sporadic, there are a number of uncommon predisposing germline mutation syndromes, e.g., Gorlin's syndrome, Turcot's syndrome, and Li-Fraumeni syndrome [2, 3].

Anhidrotic ectodermal dysplasia (AED) is a rare syndrome with an incidence of approximately 1:100,000 [4]. AED is characterized by abnormalities in the hair, teeth, nails, and sweat glands which may have been inherited as an X-linked recessive, autosomal dominant trait, or as an autosomal recessive trait [5]. Several malignant tumors, such as squamous cell carcinoma [6-8], malignant melanoma [9], rhabdomyosarcoma [10], and neuroblastoma [11], have been observed in AED patients, although AED-complicated brain tumors, such as medulloblastoma, have not been reported to date.

In this case report, we describe an AED-affected 1-yearold boy who developed a multiple desmoplastic/nodular medulloblastoma (DNMB).

\section{Case report}

A 1-year-old boy was referred to our institution because of ingravescent vomiting. He was born at term after an 
uneventful pregnancy, and his mother has peg-shaped teeth. He suffered repeatedly from upper and lower respiratory infections. He had a characteristic depressed nasal bridge, low-set ears, thick lips with peg-shaped teeth, and often had febrile episodes (Fig. 1). In addition to the aforementioned features, detailed physical examination disclosed marked sweating deficiency, sparse hair, thin and atrophic skin, and scaly dermatitis with frontal bossing. On the basis of the clinical findings, the patient was diagnosed as suffering from AED by pediatrics. His maternal grandfather also displayed peg-shaped teeth, depressed nasal bridge, low-set ears, thick lips, sparse hair, thin atrophic skin, and hypohidrosis. On admission, physical and neurological examinations further disclosed growth retardation, macrocephaly with scalp varicosis, bilateral papilledema, and disturbance of consciousness.

Computerized tomography (CT) and magnetic resonance imaging (MRI) revealed one large and two small heterogeneously enhanced posterior fossa masses with speckled calcifications and obstructive hydrocephalus (Fig. 2). Whole neural axis MRI demonstrated no other parenchymal lesions.

Because his consciousness worsened on the day after admission, tumor resection was performed by midline suboccipital craniotomy. Before craniotomy, a ventricular drainage was performed to prevent the risk of acute hydrocephalus. Because intracranial pressure was very intense, we uncapped the cerebellum surface of the tumor. After uncapping the cerebellar cortex covering the tumor, we removed a well-defined, gray-colored, elastic hard mass highly susceptible to hemorrhage located in the left cerebellar hemisphere. Total en block resection was possible because of a clear demarcating plane between the tumor and cerebellum.

Histological examination revealed the classical features of DNMB (Fig. 3): tumor consisted of densely packed highly cellular areas along with less cellular tumor nodules. The tumor cells in the nodular regions were uniform in size and shape, and proliferated within the neuropil-like background with focal necrotic foci in its center. Densely packed tumor cells in the cellular areas possessed more irregular and hyperchromatic nuclei and showed many typical and atypical mitoses. Reticulin staining showed a biphasic pattern composed of highly cellular desmoplastic regions and less cellular reticulin-free nodules.

An immunohistochemical study was subsequently performed after high temperature epitope unmasking using the Universal Immuno-enzyme Polymer (UIP) method (Histofine Simple Stain MAX-PO) with DAB as chromogen. The antibodies used were anti-synaptophysin (monoclonal, clone 27G12, 1:200 dilution, Leica Biosystems Newcastle Ltd, Newcastle Upon Tyne, UK), anti-Chromogranin A (monoclonal, Clone DAK-A3, 1:200 dilution, Dako, Glostrup, Denmark), anti-GFAP (polyclonal, 1:50 dilution, Dako), and anti-Ki67 (monoclonal, MIB-1 clone, 1:100 dilution, Immunotech, Marseille, France). The tumor cells were immunoreactive to chromogranin A, synaptophysin, GFAP, and vimentin, but were negative against neurofilaments, epithelial membrane antigen, Olig2, or S-100P (Fig. 4). Stronger immunoreactivities to chromogranin A and GFAP were observed in the desmoplastic regions, whereas intense synaptophysin reactivity was present in the nodular regions. Ki-67 labeling index was higher in the desmoplastic regions (maximum $70 \%$ ) than the nodular regions (maximum $45 \%$ ).

Postoperatively, the patient received multiple-drug chemotherapy, including auto peripheral blood stem cell transplantation. At the last follow-up 12 months after surgery, he was doing well with complete remission when evaluated with imaging studies. A genetic test was not performed because of familial refusal.
Fig. 1 Signs of sparse hair, thin atrophic skin, scaly dermatitis, and low-set ears were observed on admission (a); and the 1-year-old male patient exhibited frontal bossing, a depressed nasal bridge, thick lips with peg-shaped teeth (b). Alopecia was a side effect of multiple-drug chemotherapy
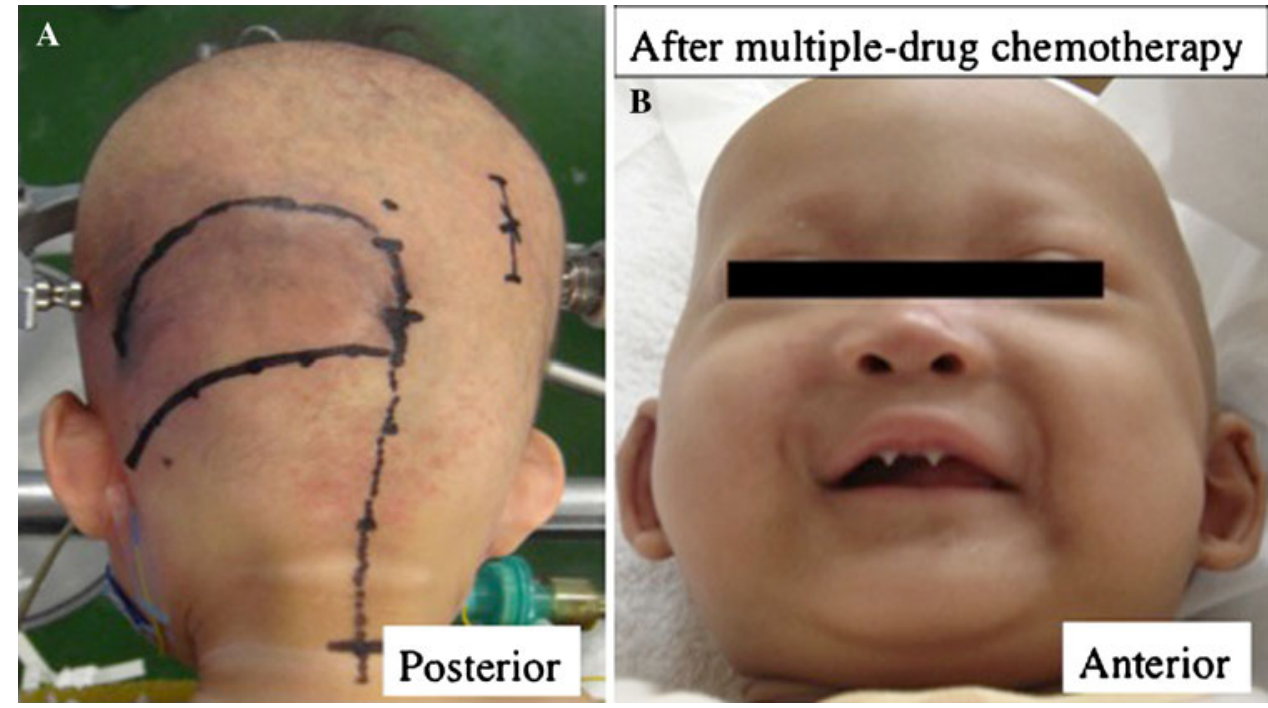
Fig. 2 Post-contrast axial (a, b), coronal (c), and sagittal (d) T1-weighted magnetic resonance images (MRI) showing one large and two small heterogeneously enhanced posterior fossa masses with obstructive hydrocephalus
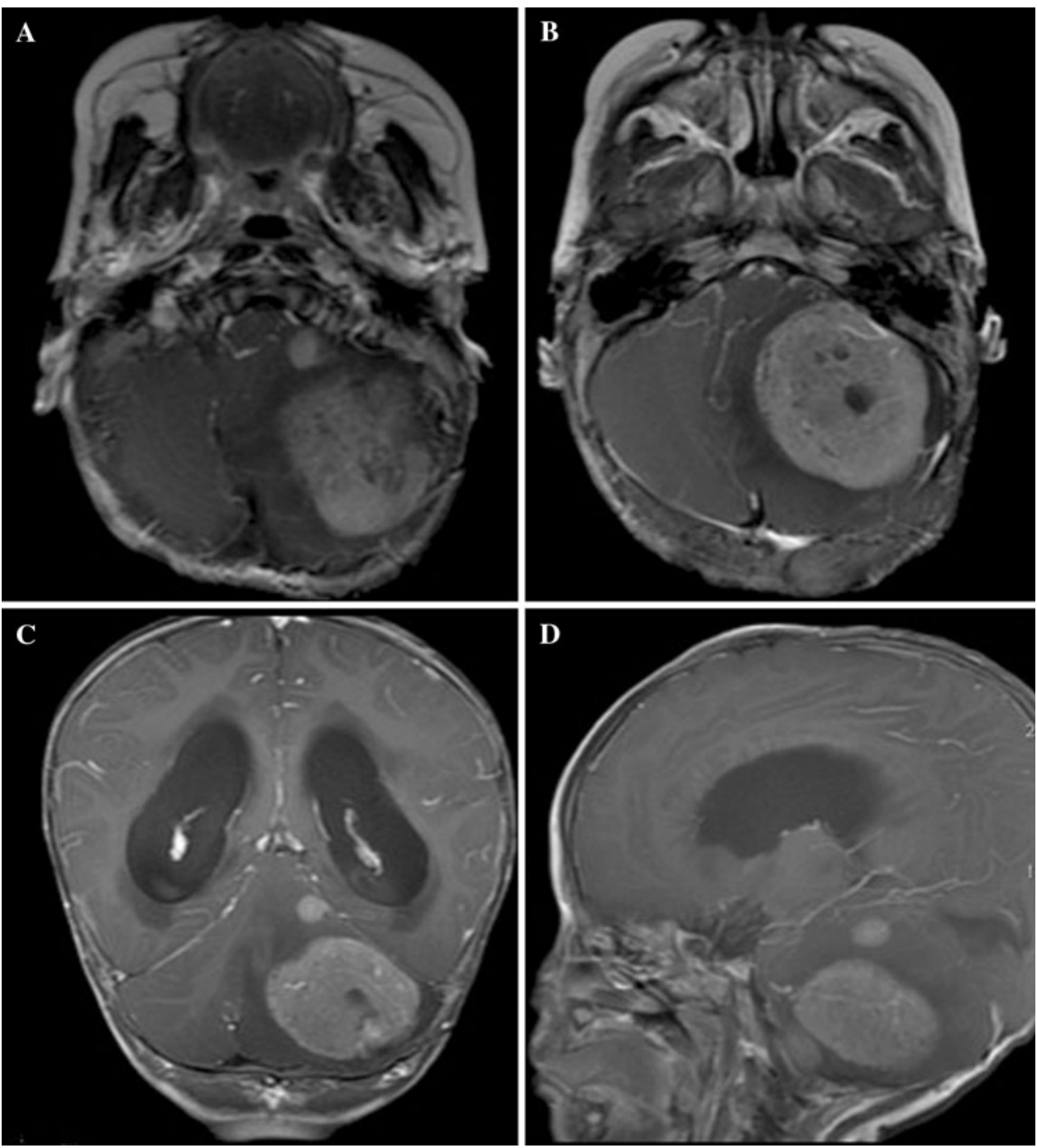

Fig. 3 The tumor was composed of densely packed cellular areas and less cellular tumor nodules. The densely packed tumor cells had irregular and hyperchromatic nuclei and many mitoses (H\&E staining: $\mathbf{a} \times 100, \mathbf{b}, \mathbf{c} \times 200)$. Reticulin stain (d) shows a biphasic pattern with reticulin-rich cellular regions and less cellular, reticulin-free nodules
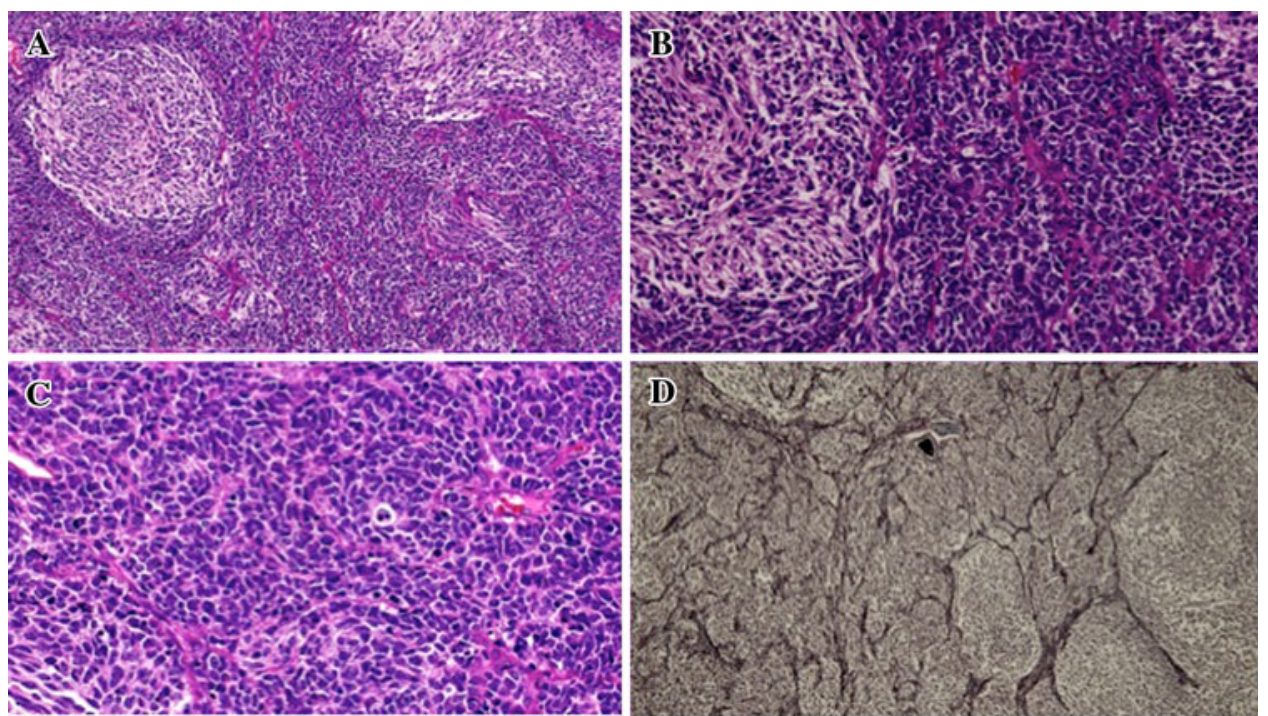


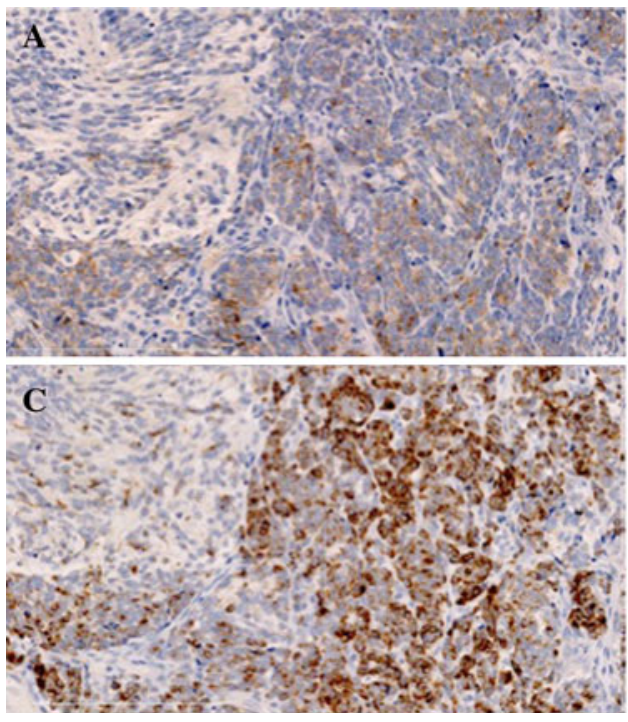

Fig. 4 The tumor cells were immunoreactive to chromgranin A, synaptophysin, and GFAP. The desmoplastic regions showed stronger chromogranin A and GFAP reactivities with less intensive synaptophysin staining, whereas the nodular regions showed stronger synaptophysin reactivity with less intensive reactivity for chromogranin A

\section{Discussion}

$\mathrm{AED}$, which is a rare syndrome, characteristically involves all the ectodermal derivatives with marked sweating deficiency. All relevant structures, such as keratinocytes, melanocytes, hair, sweat glands, sebaceous glands, nails, and teeth are defective anatomically and functionally [12]. A majority of AED patients have X-linked recessive inheritance: more than $90 \%$ of the affected individuals are male, and female carriers may show incomplete clinical features of the disease [13]. In our patient, although mutational analysis was not performed, AED was most likely due to the $\mathrm{X}$-linked recessive form, because his mother showed incomplete clinical features and his maternal grandfather exhibited the complete features of AED. Although the genetic basis of AED is not fully understood, there are currently at least two known types of AED with the $\mathrm{X}$-linked recessive form [14] on the molecular basis: (1) AED due to a mutation in the ectodysplasin A (EDA) gene located at Xq12-q13.1; and (2) AED associated with immunodeficiency involving a mutation of the nuclear factor $\mathrm{\kappa B}$ gene essential modulator (NEMO), or nomo-like kinase (NLK). AED-related tumors reported in the literature are squamous cell carcinoma of the skin [6], nail bed [7], trachea [8], malignant melanoma of the skin [9], rhabdomyosarcoma in the nasal cavity and nasopharynx [10], and neuroblastoma in the thoracic cavity [11]; however, an association of AED syndrome with brain tumors including medulloblastoma has yet to be elucidated. As far we know, this is the first report of AED-associated DNMB.

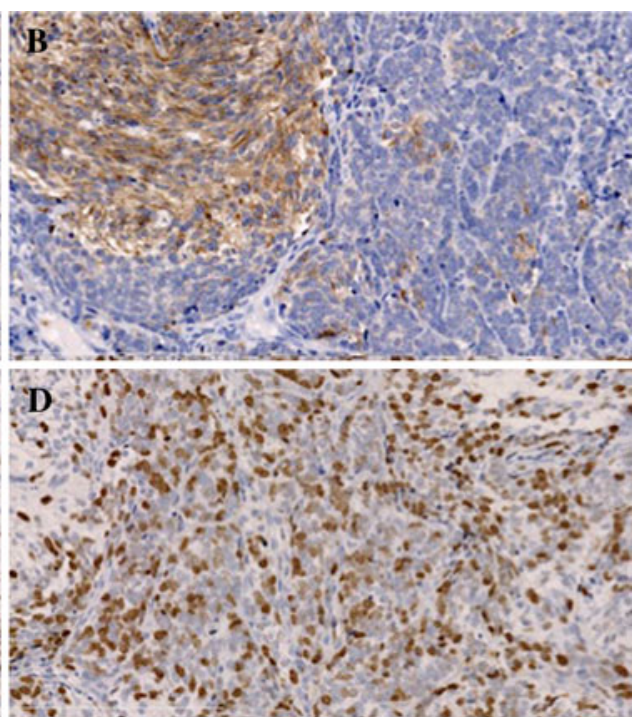

and GFAP. Neurofilaments, epithelial membrane antigen, Olig2, and S-100P were negatively stained. The MIB-1 labeling index was higher in the desmoplastic regions (maximum $70 \%$ ): a (chromogranin A), b (synaptophysin), c (GFAP), and d (Ki-67)

Approximately $10 \%$ of children with brain tumors have a genetic disorder that places them at increased risk of developing cancers [15]. Some well-described associations occur between medulloblastoma and Gorlin's syndrome, Turcot's syndrome, and Li-Fraumeni syndrome [2, 3]. In particular, medulloblastoma is found in approximately 3-5 \% of patients with Gorlin's syndrome (with germline mutation of the Hedgehog (Hh) receptor PTCH) [2], and almost all medulloblastomas associated with Gorlin's syndrome are a subtype of DNMB.

Classification schemes for medulloblastomas are based primarily on histopathology and include variants such as DNMB, medulloblastoma with extensive nodularity (MBEN), classic medulloblastoma, large cell, and anaplastic medulloblastoma. With recent developments in monitoring transcription across the genome, various groups have started to subclassify medulloblastomas on the basis of their differences in the transcriptome [16]. Four principal subgroups have been identified and clarified, i.e., Wnt, Shh, group C, and group D [16]. The Wnt and Shh groups are named according to the signaling pathways thought to play a prominent role in the pathogenesis of that subgroup [16]. Since less is known about the biology of the remaining two subgroups, a consensus to retain the generic names for the present is adopted until the underlying biology driving these subgroups can be better delineated [16]. The Shh group is named after the Hh-signaling pathway: viz., a subgroup characterized by activation of the Hh-signaling pathway occurs primarily in infants and adults, and is thought to be associated with DNMB [16]. 
The Hh-signaling pathway is of central importance during embryo development in metazoans, as it governs a diverse array of processes that involve cell proliferation, differentiation, and tissue-patterning [17]. In the development of the skin and teeth, the Hh pathway is crucial for maintaining stem cell population, regulating hair follicle and sebaceous gland development in the skin, and normal development of teeth [18]. Many congenital malformations in humans, such as holoprosencephaly, brachydactyly, and limb malformations, are known to involve mutations in various components of the Hh-signaling pathway [19]. In neoplasms, the Hh pathway is firmly linked not only to DNMB but also to basal cell carcinoma [17]. Moreover, it has been reported that other sporadic cancers, including those in the pancreas, prostate, lung, and breast, could also be dependent on the Hh pathway activity [17].

Hh-signaling also modulates the expression of X-linked inhibitor of apoptosis (XIAP), which serves to repress the type-1 death receptor pathway in human cholangiocarcinoma cells [20]. Deregulation of XIAP can result in cancer, neurodegenerative disorders, and autoimmune disorders [21]. DNMB may be associated with AED via the X-linked events between Hh-signaling and XIAP. Moreover, other medulloblastoma subtypes may be associated with $\mathrm{X}$-linked gene disruptions [22]. Future studies on the gene type of medulloblastomas and AED are necessary to understand the AED-DNMB association.

In conclusion, we encountered a very rare case of AEDassociated DNMB in a male infant. Although this hitherto unknown association could have occurred by chance, further studies on the Hh-signaling pathway may clarify this DNMB-AED association.

Acknowledgments This study was partially supported by the Japan Society for the Promotion of Grant-in-Aid for Young Scientists (B) no. 24791503 and Grants-in-Aid for Scientific Research (C) no. 22591612 and no. 23592091.

Conflict of interest The authors declare that they have no conflict of interest

\section{References}

1. Young JL Jr, Miller RW (1975) Incidence of malignant tumors in US children. J Pediatr 86:254-258

2. Evans DG, Farndon PA, Burnell LD et al (1991) The incidence of Gorlin syndrome in 173 consecutive cases of medulloblastoma. Br J Cancer 64:959-961
3. Jamjoom ZA, Sadiq S, Mofti AB et al (1989) Turcot syndrome: report of a case and review of the literature. Int Surg 74:45-50

4. Clarke A (1987) Hypohidrotic ectodermal dysplasia. J Med Genet 24:659-663

5. Priolo M, Silengo M, Lerone $M$ et al (2000) Ectodermal dysplasias: not only 'skin' deep. Clin Genet 58:415-430

6. McGregor JM, Hawk JL (1997) Increased risk of skin cancer in patients with ectodermal dysplasia - a contraindication to psoralen and UVA (PUVA) therapy? Clin Exp Dermatol 22:56

7. Mauro JA, Maslyn R, Stein AA (1972) Squamous-cell carcinoma of nail bed in hereditary ectodermal dysplasia. N Y State J Med 72:1065-1066

8. Winter SC, Bates GJ (2002) Hypohidrotic ectodermal dysplasia associated with squamous cell carcinoma of the trachea. J Laryngol Otol 116:742-743

9. Gregoriou S, Rigopoulos D, Vergou T, Korfitis C, Menegakis G, Kontochristopoulos G (2007) Should we consider hypohidrotic ectodermal dysplasia as a possible risk factor for malignant melanoma? J Cutan Med Surg 11:188-190

10. Cankaya H, Kösem M, Kiris M, Uner A, Metin A (2002) Nasopharyngeal rhabdomyosarcoma in a patient with hypohidrotic ectodermal dysplasia syndrome. Auris Nasus Larynx 29:313-316

11. Buoni S, Zannolli R, Macucci F et al (2007) Hypohidrotic ectodermal dysplasia and intrathoracic neuroblastoma. Pediatr Dermatol 24:267-271

12. Vargas GA, Fantino E, George-Nascimento C et al (1996) Reduced epidermal growth factor receptor expression in hypohidrotic ectodermal dysplasia and Tabby mice. J Clin Invest 97:2426-2432

13. Harper JI (1998) Genetics and genodermatoses. In: Champion RH (ed) Textbook of dermatology, vol 1, 6th edn. Blackwell-Science, Oxford, pp 357-436

14. Itin PH, Fistarol SK (2004) Ectodermal dysplasias. Am J Med Genet C Semin Med Genet 15:45-51

15. Blaney SM, Kun LE, Hunter J et al (2006) Tumors of the central nervous system. In: Pizzo PA (ed) Principles and practice of pediatric oncology. Lippincott Williams \& Wilkins, Philadelphia, pp 786-864

16. Northcott PA, Korshunov A, Witt H et al (2011) Medulloblastoma comprises four distinct molecular variants. J Clin Oncol 10:1408-1414

17. Teglund S, Toftgård R (2010) Hedgehog beyond medulloblastoma and basal cell carcinoma. Biochim Biophys Acta 1805:181-208

18. Athar M, Tang X, Lee JL et al (2006) Hedgehog signalling in skin development and cancer. Exp Dermatol 15:667-677

19. Nieuwenhuis E, Hui CC (2005) Hedgehog signaling and congenital malformations. Clin Genet 67:193-208

20. Kurita S, Mott JL, Cazanave SC et al (2011) Hedgehog inhibition promotes a switch from type II to type I cell death receptor signaling in cancer cells. PLoS ONE 31:e18330

21. Wilkinson JC, Cepero E, Boise LH et al (2004) Upstream regulatory role for XIAP in receptor-mediated apoptosis. Mol Cell Biol 24:7003-7014

22. Anderton JA, Lindsey JC, Lusher ME et al (2008) Global analysis of the medulloblastoma epigenome identifies disease-subgroupspecific inactivation of COL1A2. Neuro Oncol 10:981-994 